\title{
Heparin-induced thrombocytopenia in patients receiving mechanical circulatory support
}

Soren Schenk, MD, a Aly El-Banayosy, MD, ${ }^{a}$ Wolfgang Prohaska, MD, ${ }^{b}$ Latif Arusoglu, MD, a Michiel Morshuis, MD, ${ }^{a}$ Wilhelm Koester-Eiserfunke, PhD, ${ }^{\mathrm{b}}$ Lukasz Kizner, MD, ${ }^{a}$ Edward Murray, MD, ${ }^{a}$ Petra Eichler, PhD, ${ }^{\mathrm{c}}$ Reiner Koerfer, MD, and Andreas Greinacher, MD ${ }^{\mathrm{C}}$

Supplemental material is available online.
From Thoracic and Cardiovascular Surgery $^{\mathrm{a}}$ and Laboratory and Transfusion Medicine, ${ }^{\mathrm{b}}$ Heart Center North RhineWestphalia, Ruhr-Universität Bochum, Bad Oeynhausen, Germany, and Immunology and Transfusion Medicine, ${ }^{\mathrm{c}}$ Ernst-MoritzArndt University, Greifswald, Germany.

Read at the Eighty-fifth Annual Meeting of The American Association for Thoracic Surgery, San Francisco, Calif, April 10-13, 2005.

Received for publication May 2, 2005; revisions received Dec 18, 2005; accepted for publication Jan 10, 2006.

Address for reprints: Aly El-Banayosy, MD, Heart Center NRW, Georgstrasse 11, Bad Oeynhausen, Germany, 32545 (E-mail: abanayosy@hdz-nrw.de).

J Thorac Cardiovasc Surg 2006;131:1373-81 $0022-5223 / \$ 32.00$

Copyright $\odot 2006$ by The American Association for Thoracic Surgery

doi:10.1016/j.jtcvs.2006.01.048
Objectives: Patients receiving mechanical circulatory support are at risk for the development of heparin-induced thrombocytopenia due to the prolonged postoperative use of heparin. We evaluated their antibody status and outcome.

Methods: Between 2003 and 2004, 115 patients received mechanical circulatory support for more than 5 days. Blood samples from postoperative day 7 were retrospectively analyzed for anti-platelet factor 4/heparin antibodies and heparin-induced platelet activation.

Results: Overall, $12(10.6 \%)$ patients had heparin-induced thrombocytopenia as defined by in vitro platelet activation, 28 (24.8\%) had nonactivating antibodies, and $73(64.6 \%)$ were classified as negative for antibodies. Patients positive for heparininduced thrombocytopenia had the highest levels of anti-platelet factor 4/heparin immunoglobulin $\mathrm{G}$ antibodies. Freedom from thromboembolism was 33\%, 33\%, and $16 \%$ at 1,3 , and 6 months for positive patients, $77 \%, 68 \%$, and $55 \%$ for negative patients $(P<.001)$, and $70 \%, 53 \%$, and $53 \%$ for patients with nonactivating antibodies $(P=.068)$, respectively. The relative risk for thromboembolism in antibody positive patients peaked in the first month of support (odds ratio 7.46, $P=$ .002). Independent risk factors for thromboembolic events included higher antiplatelet factor 4/heparin antibody titers, female gender, and higher fibrinogen levels.

Conclusion: Heparin-induced thrombocytopenia was more prevalent in patients receiving mechanical circulatory support than in other cardiac patients. Frequent antibody screening is recommended due to the increased risk of thromboembolism. Heparin alternatives should be subjected to clinical trials in these high-risk patients.

$\mathrm{H}$ eparin is a standard anticoagulant, but adverse events associated with its use are frequent. Antibodies can be formed against complexes of platelet factor 4 (PF4), a self protein, and heparin. The resulting PF4/heparin/immunoglobulin $\mathrm{G}(\mathrm{IgG})$ complexes then induce platelet activation, which, in turn, results in arterial and venous thrombus formation. ${ }^{1,2}$ Anti-PF4/heparin antibodies are detected in more than $50 \%$ of patients undergoing cardiac surgery, ${ }^{3-6}$ although the clinical relevance remains debated. Only $1 \%$ to $3 \%$ of patients are reported to have clinically relevant heparin-induced thrombocytopenia (HIT), ${ }^{4,5}$ a syndrome based on clinical and laboratory signs: that is, thromboembolism, decrease of platelet counts, and anti-PF4/heparin antibodies. ${ }^{7}$ However, additional consideration is warranted in patients receiving mechanical circulatory support (MCS). These patients are commonly exposed to prolonged use of heparin before surgery and, unlike other cardiac patients, invariably receive heparin after surgery for several weeks. The immune response leading to anti-PF4/heparin antibody formation may thus be induced for a longer biologically active time. Subsequently, HIT antibodies may even act in concert with other thrombogenic factors inherent to mechanical support, 


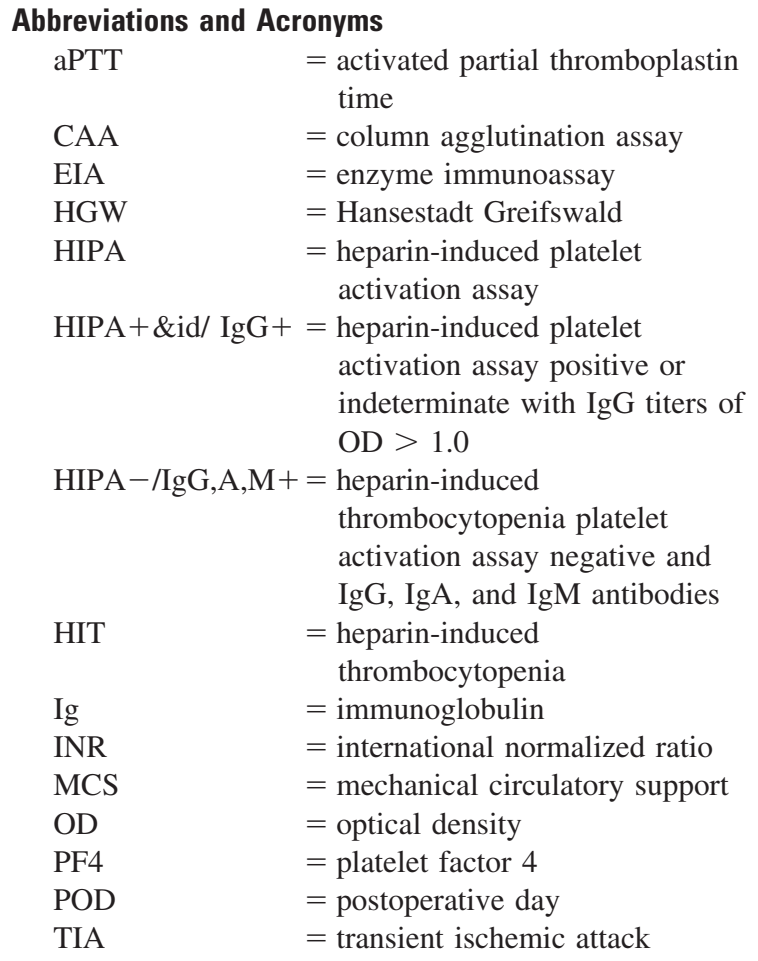

such as platelet and coagulation activation. Selected studies suggest that this may indeed be true, but a detailed assessment of the interrelationship between platelet counts, anti-PF4/heparin antibodies, coagulation monitoring, and clinical events has not been performed.

To better manage these critically ill patients by choosing appropriate anticoagulation, we sought to test our clinical observation that HIT is an underappreciated problem with therapeutic challenges and serious consequences. Thus, for patients undergoing MCS, we sought to (1) evaluate prevalence, amount, and Ig classes of anti-PF4/heparin antibodies, (2) provide an assessment of heparin-induced platelet activation to diagnose or rule out HIT, and (3) determine the impact of anti-PF4/heparin antibodies on clinical outcomes.

\section{Methods}

\section{Patients and Devices}

From January 2003 to December 2004, 126 patients were placed on MCS at the Heart Center NRW Bad Oeynhausen, Germany, using 10 different left ventricular or biventricular devices (Appendix E1). Device selection criteria and management have been published previously. ${ }^{8}$ Patients with a support time of 5 days or fewer because of death due to circulatory collapse $(n=10)$ or heart transplantation $(\mathrm{n}=1)$ were not included in this study, leaving 115 patients. Patient characteristics are summarized in Table E1. Data were extracted from the institutional data base endorsed by The Institutional Review Board of the Heart Center, Bad Oeynhausen, Germany.
Thromboembolism was confirmed or excluded on clinical status by computed tomography, angiography, and/or Doppler ultrasonography. Patients who died while receiving MCS underwent autopsy by a pathologist experienced in cardiovascular diseases and devices.

\section{Anticoagulation Protocol and Blood Sampling}

All patients were receiving heparin before surgery, and all patients had a likely history of heparin exposure during previous hospitalizations. Only unfractionated heparin obtained from porcine intestinal mucosa was used. Assist devices were implanted during cardiopulmonary bypass with heparin used for anticoagulation (activated clotting time $>400$ seconds). Heparin was reversed by protamine and then withheld for 24 hours or as long as chest tube drainage remained greater than $100 \mathrm{~mL} / \mathrm{h}$. Thereafter, all patients received heparin for several weeks postoperatively (activated partial thromboplastin time [aPTT] 40-60 seconds) and were then switched to oral phenprocoumon (international normalized ratio [INR] 2.5-3.5). Antiplatelet agents, that is, aspirin or clopidogrel, were given in selected patients beginning not before the second postoperative month.

Blood samples were obtained daily for platelet counts and standard coagulation assays, including aPTT, INR, fibrinogen, and D-dimers. Tests for the presence of anti-PF4/heparin antibodies were ordered in cases of suspected HIT at the attending physician's discretion. Indications were otherwise unexplained thrombocytopenia and/or the occurrence of thromboembolism. Additional blood samples were collected on postoperative days (POD) 5 to 7 in citrated tubes, centrifuged, and stored at $-80^{\circ} \mathrm{C}$. These samples were used for retrospective tests of anti-PF4/heparin antibodies and heparin-induced platelet activation. All samples were obtained from an arterial line after $10 \mathrm{~mL}$ of blood was withdrawn from the line.

\section{Anti-PF4/Heparin Antibodies}

The presence of antibodies to the heparin/PF4 platelet protein complex was tested by the polyspecific enzyme immunoassay GTI-PF4 (EIA GTI) (Genetic Testing Institute, Waukesha, Wis) with an optical density (OD) of 0.5 as cutoff point. All stored blood samples from PODs 5 to 7 were also tested by EIA GTI and additionally by the column agglutination assay (CAA) (DiaMed Diagnostika, Bensheim, Germany). The latter test provides a semiquantitative signal ranging from 0 (no antibodies detected) to 3 (highest level of particle agglutination). EIA GTI and/or CAApositive blood probes were sent blinded to a reference laboratory for evaluation by custom-made mono-specific EIA (EIA Hansestadt Greifswald [HGW]) for Ig classes and by heparin-induced platelet activation assay (HIPA), as previously described. ${ }^{9}$ The combined use of HIPA with mono-specific EIA was validated for diagnosing HIT in previous studies. ${ }^{10}$ Specifically, antibodies testing positive by the HIPA were considered clinically relevant HIT antibodies. If the HIPA showed heparin-independent platelet activation, the test was designated indeterminate. Only in those cases, an $\operatorname{IgG}$ class antibody titer of OD greater than 1.0 defined clinically relevant HIT antibodies. Conversely, HIPA negative antibodies were considered clinically not relevant regardless of their $\operatorname{IgG}$ titer. Finally, all $\operatorname{IgA}$ and $\operatorname{IgM}$ antibodies were considered irrelevant. 


\section{3-2004 Patients on MCS >5 days $n=115$}

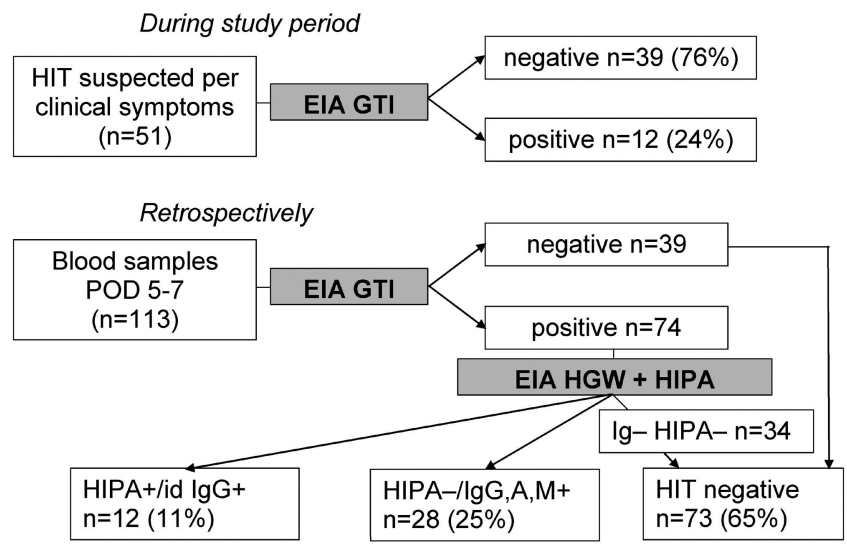

Figure 1. Sequence of anti-PF4/heparin antibody testing. MCS, mechanical circulatory support; EIA GTI, polyspecific anti-PF4/ heparin antibody enzyme immunoassay; EIA HGW, monospecific anti-PF4/heparin antibody enzyme immunoassay; HIPA, heparininduced platelet activation assay; HIT, heparin-induced thrombocytopenia; POD, postoperative day; Ig, immunoglobulin.

\section{Data Analysis}

Data are presented as percentage, mean \pm standard deviation, or 95\% confidence interval. Several demographic, procedural, and laboratory variables were considered as potential risk factors for thrombotic events (Appendix E2). Univariate comparisons were made by the Mann-Whitney test, Student $t$ test, Kendall tau, $\chi^{2}$ test, or 1-way analysis of variance with Bonferroni post-hoc testing, as appropriate. Those factors with a statistically significant difference $(P<.10)$ were then included stepwise (forward and backward) in a multivariate logistic regression with thrombotic event as the dependent variable. The model was built with 64 randomly selected patients and then validated on the remaining 51 patients. Variables were retained in the model at a significance level of $P<.05$. The distribution of clinical events over time was analyzed by nonparametric Kaplan-Meier estimates, followed by a log-rank test. Clinical events per time interval were compared with the $\chi^{2}$ test, and their relative risk was calculated. Predictive capabilities of various anti-PF4/heparin antibody tests with respect to the occurrence of thromboembolism were estimated by Bayesian reasoning. All analyses were performed with SPSS 12.0 (SPSS Inc, Chicago, Ill).

\section{Results \\ Anti-PF4/Heparin Antibodies}

Complete information on the presence of anti-PF4/heparin antibodies by POD 7 was available in 113 of 115 patients; all of them at least briefly received heparin after surgery. At POD 7, 39 of 113 (34.5\%) blood probes tested negative by EIA GTI and CAA, including 5 patients subsequently becoming EIA GTI positive (PODs 12, 14, 18, 30, and 240, respectively). Seventy-four $(65 \%)$ tested positive for antiPF4/heparin antibodies by EIA GTI and/or CAA. These samples were further tested by mono-specific EIA HGW and HIPA. Overall, a group of $12(10.6 \%)$ patients either had a positive HIPA test $(\mathrm{n}=10)$ and anti-PF4/heparin IgG antibodies or an indeterminate HIPA test and anti$\mathrm{PF}$ 4/heparin $\operatorname{IgG}$ antibodies with an OD greater than 1.0 $(\mathrm{HIPA}+\& \mathrm{id} / \mathrm{IgG}+)$. Another $28(24.8 \%)$ patients were HIPA negative but had anti-PF4/heparin antibodies of $\mathrm{IgG}$, IgA, or IgM classes (HIPA - /gG,A,M+). A third group of $73(64.6 \%)$ patients were anti-PF4/heparin antibody negative (Figure 1, Table 1). Anti-PF4/heparin IgA and IgM levels did not differ significantly between HIPA+\&id/ IgG + and HIPA-/IgG,A,M+ patients (Table 1).

\section{Clinical Diagnosis and Treatment of Heparin-Induced Thrombocytopenia} In 51 of 115 patients, anti-PF4/heparin antibodies were assessed at the attending physician's discretion to diagnose or to rule out HIT (Figure 1). Tests were ordered at a median of 5 days (range 26-279 days), predominantly due to a low platelet count for at least 2 days $(76,000 / \mathrm{mL} \pm 52,000 / \mathrm{mL})$. In one case, a thromboembolic event (limb ischemia) preceded assessment of antibody status. Of note, the majority of tests for anti-PF4/heparin antibodies were ordered in the second half of the study period ( 38 vs 13 cases), likely due to an enhanced awareness of HIT.

Among 51 patients tested for HIT, 12 patients (24\%) had anti-PF4/heparin antibodies by the polyspecific EIA GTI during their hospital stay. Of these, 6 patients were retrospectively confirmed by $\mathrm{HIPA}+\& \mathrm{id} / \mathrm{IgG}+$. One patient

TABLE 1. Amount and Ig classes of anti-PF4/heparin antibodies and heparin-induced platelet activation

\begin{tabular}{llllll}
\hline & \multicolumn{1}{c}{ EIA GTI (OD) } & \multicolumn{1}{c}{ IgG (OD) } & \multicolumn{1}{c}{ IgA (OD) } & IgM (OD) & HIPA (pos/ neg) \\
\hline HIPA+\&id/lgG+ & $1.810 \pm 0.91^{*} \dagger(\mathrm{n}=12)$ & $1.508 \pm 0.41^{*} \dagger(\mathrm{n}=12)$ & $0.872 \pm 0.65 \dagger(\mathrm{n}=12)$ & $0.611 \pm 0.41 \dagger(\mathrm{n}=12)$ & $10 / 2(\mathrm{n}=12)$ \\
HIPA-/IgG,A,M+ & $0.852 \pm 0.52(\mathrm{n}=28)$ & $0.652 \pm 0.39(\mathrm{n}=28)$ & $0.543 \pm 0.45(\mathrm{n}=28)$ & $0.397 \pm 0.29(\mathrm{n}=28)$ & $0 / 28(\mathrm{n}=28)$ \\
HIT negative & $0.397 \pm 0.31(\mathrm{n}=73)$ & $0.244 \pm 0.12(\mathrm{n}=34)$ & $0.182 \pm 0.09(\mathrm{n}=34)$ & $0.201 \pm 0.08(\mathrm{n}=34)$ & $0 / 39(\mathrm{n}=34)$ \\
\hline
\end{tabular}

EIA GTI (OD), Titer of anti-platelet factor 4/heparin antibodies by polyspecific enzyme immunoassay; OD, optical density; Ig, immunoglobulin; HIPA, status of heparin-induced platelet activation (yes/no); HIPA+\&id/IgG+, heparin-induced platelet activation assay positive or indeterminate with IgG titers of $\mathrm{OD}>1.0 ; \mathrm{HIPA}-/ \lg G, A, M+$ heparin-induced thrombocytopenia platelet activation assay negative and $\operatorname{IgG}, \operatorname{IgA}$, and IgM antibodies; HIT, heparin-induced thrombocytopenia. *HIPA+\&id/lgG + versus HIPA-/IgG,A,M+,P<.001. †HIPA+\&id/lgG + versus HIT negative, $P<.001$. 

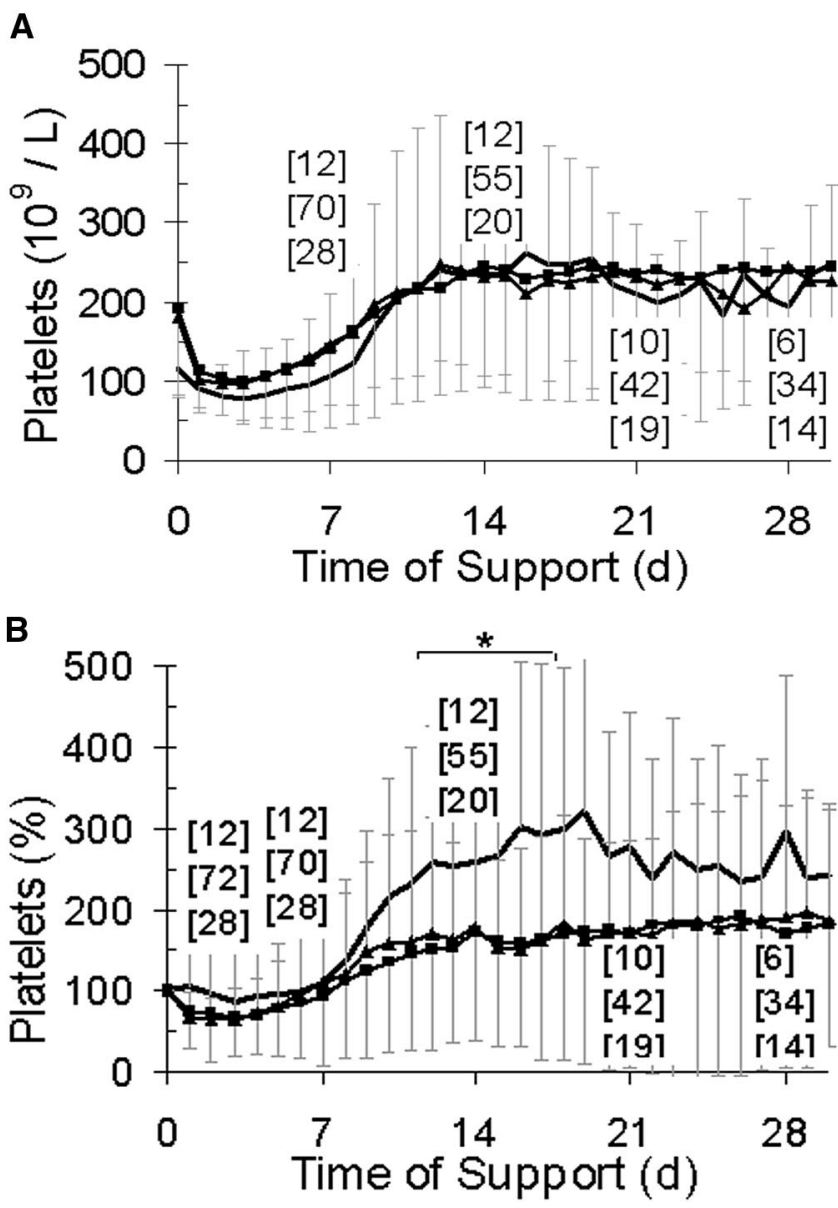

Figure 2. A, Platelet counts during mechanical support. B, Relative platelet counts (percent change from baseline) during mechanical support. Numbers in parentheses indicate patients for whom data are available. Vertical bars indicate 1 SD. Solid line (-), Heparin-induced platelet activation assay positive or indeterminate with IgG titers of $\mathrm{OD}>1.0$; solid line with squares $(-1)$, heparin-induced thrombocytopenia negative; solid line with triangles (- - ), heparin-induced thrombocytopenia platelet activation assay negative and $\lg G, \lg A$, or $\lg M$ antibodies. ${ }^{*} P<.05$.

emerged as $\mathrm{HIPA}-/ \mathrm{IgG}, \mathrm{A}, \mathrm{M}+$ and 5 patients were found to be HIT negative.

Heparin was suspended in 12 patients once they were found positive by the EIA GTI, and lepirudin or antiplatelet acetylsalicylic acid and/or epoprostenol was substituted. Despite alternative anticoagulation, all 6 patients later confirmed as HIPA+\&id/IgG + had thromboembolic events (Table E2). The 1 patient with HIPA-/IgG,A,M+ status had cerebral bleeding (POD 9) while receiving lepirudin, and 1 HIT negative patient receiving epoprostenol had a cerebral transitory ischemic attack (TIA) with no residuals (POD 8). Furthermore, 1 HIT negative patient had a TIA long after epoprostenol treatment while receiving phenproc-

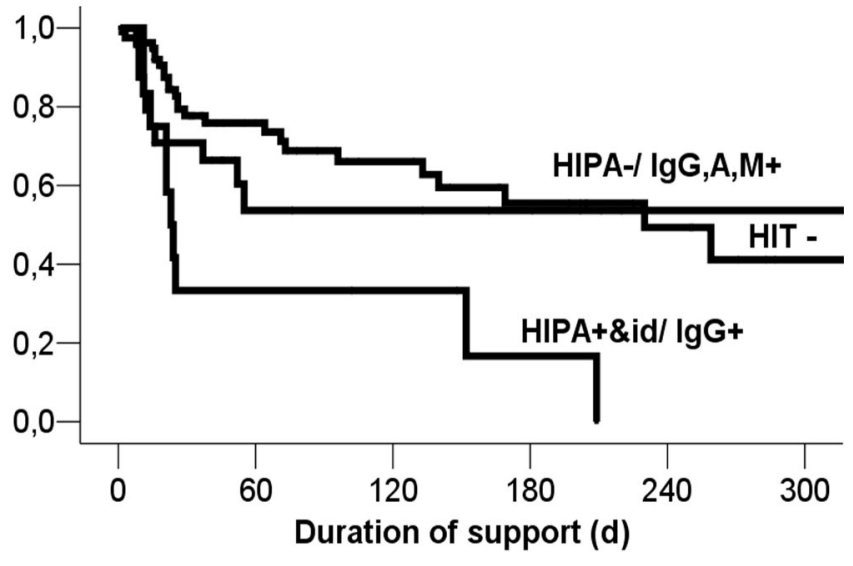

Figure 3. Freedom from thromboembolic events during mechanical support. HIPA+\&id/IgG+, Heparin-induced platelet activation assay positive or indeterminate with $\lg G$ titers of $O D>1.0$; HIT-, heparin-induced thrombocytopenia negative; HIPA-/ $\lg G, A, M+$, heparin-induced thrombocytopenia platelet activation assay negative and $\lg G, \lg A$, or $\lg M$ antibodies.

oumon (POD 133) and 3 HIT negative patients remained free of thromboembolism.

Platelet Counts and Standard Coagulation Monitoring There was a transient drop in platelet count until POD 7 in all groups, and absolute platelet counts recovered with no further period of thrombocytopenia thereafter (Figure 2, A). Interestingly, when computed as percent change from baseline, HIPA $+\& \mathrm{id} / \mathrm{IgG}+$ patients revealed 3 -fold higher relative platelet counts between POD 10 and POD 25 as compared with HIT negative or HIPA-/IgG,A,M+ patients (Figure 2, B).

Standard coagulation tests during MCS did not provide conclusive evidence that blood coagulation differed with respect to the anti-PF4/heparin antibody status (Table E3). There was a trend toward higher levels of D-dimers between PODs 5 to 7 in HIPA+\&id/IgG+ patients, indicating their hypercoagulability (HIPA + \&id/IgG+, HIPA-/IgG,A,M + , and HIT negative; $1183 \pm 975,1460 \pm 1631$, and $843 \pm 837$, respectively; $P=.042$ ). However, sufficient data for a later time point were not available. Furthermore, aPTT and INR were similar in all groups (Table E3).

Thromboembolic Events and Survival

Timing and frequency of thromboembolic complications differed substantially across the groups. HIPA $+\& \mathrm{id} / \mathrm{IgG}+$ patients had frequent thromboembolic events occurring predominantly in the first postoperative month (Table E2). Freedom from total thromboembolic events, including cerebrovascular accidents, TIAs, and peripheral thromboembolism, was $33 \%, 33 \%$, and $16 \%$ at 1,3 , and 6 months for 
TABLE 2. Potential risk factors associated with thrombotic events during MCS*

\begin{tabular}{lccc}
\hline \multirow{2}{*}{ Variable } & \multicolumn{2}{c}{ Thrombotic eventt } & \\
\cline { 2 - 3 } & Yes & No & $\boldsymbol{P}$ \\
\hline EIA GTI (pos/neg) & $26 / 19$ & $19 / 51$ & .003 \\
EIA GTI (OD) & $0.91 \pm 0.82$ & $0.48 \pm 0.39$ & .001 \\
HIPA (yes/no) & $9 / 36$ & $1 / 69$ & .006 \\
Gender (female/male) & $13 / 32$ & $9 / 61$ & .051 \\
Novacor device (yes/no) & $11 / 34$ & $8 / 62$ & .077 \\
aPTT (s) & $46.7 \pm 7.0$ & $50.4 \pm 8.2$ & .016 \\
Fibrinogen (mg/dL) $\ddagger$ & $572.0 \pm 139.6$ & $524.0 \pm 121.7$ & .055
\end{tabular}

EIA GTI (pos/neg), Status of anti-platelet factor $4 /$ heparin antibody by polyspecific enzyme immunoassay (positive/negative); EIA GTI (OD), titer of anti-platelet factor 4 /heparin antibodies by polyspecific enzyme immunoassay; HIPA, status of heparin-induced platelet activation (yes/no); aPTT, activated partial thromboplastin time. *See Appendix E2 for all variables tested. $\uparrow$ Thrombotic events until postoperative day 60 . $¥$ Mean over 30 days postoperatively.

HIPA $+\& \mathrm{id} / \mathrm{IgG}+$ patients, $77 \%, 68 \%$, and $55 \%$ for HIT negative (HIPA $+\& \mathrm{id} / \mathrm{IgG}+$ vs negative, $P<.001)$, and $70 \%, 53 \%$, and $53 \%$ for $\mathrm{HIPA}-/ \mathrm{IgG}, \mathrm{A}, \mathrm{M}+$ patients (HIPA +\&id/IgG + vs HIPA-/IgG,A,M,$+ P=.068$; HIPA-/IgG,A,M+ vs HIT negative, $P=.354$ ), respectively (Figure 3 ). Of note, 9 of 10 patients with a positive HIPA test developed new thrombotic complications. Further, the relative risk of thromboembolic events in HIT positive patients peaked during the first month of MCS $(\mathrm{HIPA}+\& \mathrm{id} / \mathrm{IgG}+$ vs negative and HIPA-/IgG,A,M + , odds ratio $7.46 ; P=.002$ ).

Potential risk factors for thrombotic events until POD 60 are listed in Table 2. Higher titers of anti-PF4/heparin antibodies, female gender, and higher mean fibrinogen levels were independently associated with thrombotic events (Table 3). Diagnosis of "HIPA +\&id/IgG+" cross-correlated with higher titer of anti-PF4/heparin antibodies $\left(R^{2}=\right.$ $0.690 ; P<.001)$ and was not simultaneously included in the multivariate regression model. Validation of the model showed adequate fit (Hosmer-Lemeshow test; $P=.796$ ) and satisfactory overall and negative predictive values (Table E4, Figure E1). Interestingly, no association was found between thrombotic events and device type, amount of chest tube drainage, or transfusion of packed red blood cells, platelets, and fresh frozen plasma.

The predictive capabilities of various anti-PF4/heparin antibody tests with respect to the occurrence of thromboembolic events until POD 60 were investigated. The combination of EIA GTI and CAA showed satisfactory sensitivity yet lacked specificity (Table E5). Higher titers of anti-PF4/heparin antibodies by EIA GTI increased specificity at the expense of sensitivity. In contrast, the HIPA assay in anti-PF4/heparin antibody positive patients yielded a positive predictive value of 90\% (Table E5). As expected,
TABLE 3. Factors independently associated with thrombotic events*

\begin{tabular}{lcc}
\hline Factor & Regression coefficient B (SE) & $\boldsymbol{P}$ \\
\hline Higher EIA GTI (OD) & $2.454(0.682)$ & .001 \\
Female gender & $4.292(1.228)$ & .001 \\
Higher fibrinogent & $.009(.004)$ & .007
\end{tabular}

EIA GTI (OD), Titer of anti-platelet factor 4/heparin antibodies by polyspecific enzyme immunoassay; $O D$, optical density; $S E$, standard error. *Thrombotic events until postoperative day 60 . †Mean over 30 days postoperatively.

sensitivity remained low in this setting, indicating an impact of additional thrombogenic factors other than HIT during MCS.

The frequent and earlier thromboembolic events, however, did not decrease survival. Freedom from death and transplantation at 1,3 , and 6 months was $83 \%, 58 \%$, and $33 \%$, respectively, in $\mathrm{HIPA}+\& \mathrm{id} / \mathrm{IgG}+$ patients, $68 \%$, $42 \%$, and $27 \%$, respectively, in HIT negative patients (HIPA $+\& \mathrm{id} / \mathrm{IgG}+$ vs negative, $P=.67$.$) , and 78 \%, 42 \%$, and $27 \%$, respectively, in $\mathrm{HIPA}-/ \mathrm{IgG}, \mathrm{A}, \mathrm{M}+$ patients $(\mathrm{HIPA}+\& \mathrm{id} / \mathrm{IgG}+$ vs HIPA-/IgG,A,M,$+ P=.81) . \mathrm{Sim}-$ ilar results were obtained for death or transplantation alone (data not shown).

\section{Discussion}

As demonstrated quantitatively in this study, patients receiving MCS are more prone to the development of clinical HIT than other cardiac patients. Higher titers of anti-PF4/heparin antibodies were independently associated with thromboembolic events. Heparin-induced platelet activation and the expression of anti-PF4/heparin IgG class antibodies identified patients with an increased risk for new thromboembolic complications.

\section{Anti-PF4/Heparin Antibodies During MCS}

Anti-PF4/heparin antibodies are frequently observed in patients undergoing cardiac surgery, but seroconversion was not associated with increased risk of thromboembolism in a prior study. ${ }^{3}$ HIT develops in only $1 \%$ to $3 \%$ of patients after cardiac surgery despite their high rate of seroconversion. ${ }^{5}$ Selected studies explain this discrepancy by the avoidance of postoperative heparin administration. ${ }^{3}$ However, other studies report the postoperative thrombosis rate to remain low despite postoperative heparin treatment. ${ }^{5}$ In patients receiving MCS, as many as $60 \%$ were found to be anti-PF4/heparin antibody positive. ${ }^{11}$ Interestingly, about one third of anti-PF4/heparin antibody positive patients had a thromboembolism, yet the authors did not note any thrombotic event in patients without anti-PF4/heparin antibodies. ${ }^{11}$ In contrast, our study clearly documents that the patients without PF4/heparin antibodies were at risk for 
new thrombotic complications, although the risk was 7-fold lower than in antibody-positive patients. Importantly, platelets and coagulation systems during MCS are activated by the underlying heart failure with impairment of other organ systems as well as by the large prosthetic surfaces of the devices. ${ }^{12,13}$ The contribution of these factors may in turn explain our finding that clinical HIT occurs in as many as $11 \%$ of patients receiving MCS. Of note, the diagnosis of clinical HIT was vigorously substantiated by the reference laboratory remaining blinded to the clinical course of the patients, showing heparin-induced platelet activation ${ }^{10}$ and expression of IgG classes of anti-PF4/heparin antibodies. ${ }^{14}$ Furthermore, the high number of HIT positive patients receiving MCS may be paralleled by patients undergoing heart transplantation. Indeed, Hourigan and associates ${ }^{15}$ reported 11 of 46 transplant patients having clinical HIT. Although this report has to be interpreted with caution as the study was not supported by platelet functional tests or $\operatorname{IgG}$ class antibody titers, together with our study it indicates that the manifestation of clinical HIT could be pronounced in major invasive cardiac procedures such as MCS and heart transplantation. Finally, our data are in agreement with studies of orthopedic patients, showing that the quantity of anti-PF4/heparin antibodies correlated with the magnitude of in vivo coagulation and incidence of thromboembolism. ${ }^{16,17}$ The higher the titer of anti-PF4/heparin antibodies, the more likely were our patients to have thrombotic events.

The expression of anti-PF4/heparin antibodies of less pathogenic potential, classified as HIPA-/IgG,A,M+, is another issue clarified by our work. These antibodies were found in $25 \%$ of patients and had no platelet-activating properties. The lack of platelet activation may in turn explain the similarly low risk of thromboembolism as observed in anti-PF4/heparin antibody negative patients. Thus, differentiation between clinically relevant anti-PF4/heparin antibodies ("HIT antibodies") and those with minor pathogenic potential will become essential for patient management. Since the commercially available EIAs do not distinguish between Ig classes, our study additionally explains why some patients with a positive EIA result can develop thromboembolism whereas others do not. Future investigations may focus on whether these antibodies can transform into "true" HIT antibodies on continuous heparin exposure.

\section{Platelet Counts}

Our finding that platelet counts did not differ across all groups deserves consideration. Whereas the drop of platelets until POD 5 was likely due to the use of cardiopulmonary bypass, ${ }^{5}$ thereafter, platelet count profiles were influenced by platelet damage during MCS. ${ }^{12,13}$ Frequent platelet transfusion may have augmented platelet counts and thus prevented a second drop of platelet counts after POD 5 that would otherwise have indicated development of HIT antibodies. ${ }^{4}$ Furthermore, since most patients were exposed to heparin treatment during their moribund illness, anti-PF4/ heparin antibodies may have been preformed. These preformed antibodies may in turn induce "rapid-onset HIT" on repeat heparin exposure, also leading to an early drop of platelets. ${ }^{18}$ These possibilities might also explain the relative increase of platelet counts as percent change from baseline after POD 10 in HIPA+\&id/IgG+ patients. Indeed, the platelet count profiles indicate an increased platelet turnover in HIT patients possibly due to elevated thrombopoietin levels. Additionally, the more pronounced increase of relative platelet counts in HIPA $+\& i d / \operatorname{IgG}+$ patients may be consistent with the model of increased intravascular platelet activation by pathologically relevant HIT antibodies. Nevertheless, platelet counts were not found indicative of HIT in patients receiving MCS. It is an evolving concept that HIT can manifest with a new thrombotic complication without a major decrease in platelet count, as has been shown by a retrospective analysis of 408 HIT patients ${ }^{19}$ and by a prospective cohort study. ${ }^{20}$

\section{Implications}

These findings have important implications for the management of patients receiving MCS and suggest that monitoring of anti-PF4/heparin antibodies may be relevant to identifying patients with increased risk of thrombotic events. Thromboembolism, for example, cerebrovascular accidents, is a common issue with an incidence of $6 \%$ to $30 \%$. $^{8,21,22}$ The current study demonstrates that the risk of early thromboembolic events was 7-fold higher in anti-PF4/heparin antibody positive patients and related to anti-PF4/heparin antibody titers. Thromboembolism, while nonfatal in most cases, was likely to affect morbidity, quality of life, and costs. Therefore, routine preoperative and postoperative antibody screening by polyspecific EIA and/or CAA is recommended. Patients without anti-PF4/heparin antibodies have a very low likelihood of HIT-associated thromboembolism due to the great sensitivity and negative predictive value of the tests. However, patients with anti-PF4/heparin antibodies should undergo additional assessment of Ig classes as well as platelet activating properties. This approach enhances diagnostic specificity and positive predictive value for new thromboembolic events. We now introduce the HIPA for those purposes, although this complex test requires strict quality control measures. ${ }^{23}$ Alternatively, the serotonin-release assay may be used to confirm heparininduced platelet activation with similar sensitivity and specificity. ${ }^{6,9,24}$ However, this test requires the use of radioactive tracers and, therefore, is mostly abandoned in our institutions.

Current heparin alternatives, that is, lepirudin, danaparoid and argatroban, bear substantial risk of bleeding due to 
lack of antagonists and difficulties in monitoring. ${ }^{25}$ Since only about 1 of 3 patients with a positive EIA result had indeed clinically relevant HIT antibodies, stratification according to anti-PF4/heparin Ig classes and HIPA is necessary before heparin is discontinued. Bivalirudin, another direct thrombin inhibitor and now in clinical trials, ${ }^{26}$ may be a promising alternative due to its short-acting mechanism by blood protease cleavage. Further investigations are required to assess the safety and efficacy of these heparin alternatives in patients receiving MCS.

\section{Limitations}

Blood samples for detailed analyses of anti-PF4/heparin antibodies were available only at PODs 5 to 7 . Thus, we were unable to investigate the kinetics of antibody production and seroconversion over time. Anti-PF4/heparin antibodies may be expressed only transiently, ${ }^{18}$ and patients whose antibodies disappeared after POD 7 should be at a lower risk of thromboembolism. In contrast, those patients in whom antibody titers were detectable only after POD 7 are likely to have a higher risk of thrombotic events. Finally, since only frozen samples were available for detailed analyses of anti-PF4/heparin antibodies, test sensitivities of EIA HGW and HIPA may have been too low in some cases. Thus, the relevance of HIT antibodies may have even been underestimated as compared with using fresh blood samples.

\section{Conclusion}

HIT antibodies were highly prevalent in patients receiving MCS and associated with thromboembolism. In addition to routine preoperative and postoperative antibody screening, we recommend platelet activation assays and assessment of $\mathrm{IgG}$ class antibody status. Alternative anticoagulants for patients receiving MCS are necessary so that outcomes can be improved by preventing heparin-induced complications.

We thank Marlen Ewald, BS (Bad Oeynhausen, Germany), Ulrike Strobel, BS, Birgit Fürll, BS, and Carmen Blumentritt, BS (Greifswald, Germany), for superb technical assistance. The Ventricular Assist Device Clinical Team, especially Peter Sarnowski, RN, Daniela Röfe, RN, and Frank Jaschke, RN, provided outstanding care of the patients included in this study. We additionally thank Kiyotaka Fukamachi, MD, PhD, for reviewing the manuscript and Michael Kopcak, Jr, BA (both at The Cleveland Clinic Foundation, Cleveland, Ohio), for editorial advice.

\section{References}

1. Kelton JG. The pathophysiology of heparin-induced thrombocytopenia: biological basis for treatment. Chest. 2005;127:9S-20S.

2. Warkentin TE. Heparin-induced thrombocytopenia: pathogenesis and management. Br J Haematol. 2003;121:535-55.

3. Bauer TL, Arepally G, Konkle BA, Mestichelli B, Shapiro SS, Cines DB, et al. Prevalence of heparin-associated antibodies without thrombosis in patients undergoing cardiopulmonary bypass surgery. Circulation. 1997;95:1242-6.
4. Pouplard C, May MA, Regina S, Marchand M, Fusciardi J, Gruel Y. Changes in platelet count after cardiac surgery can effectively predict the development of pathogenic heparin-dependent antibodies. $\mathrm{Br} J$ Haematol. 2005;128:837-41.

5. Warkentin TE, Greinacher A. Heparin-induced thrombocytopenia and cardiac surgery. Ann Thorac Surg. 2003;76:2121-31.

6. Warkentin TE, Sheppard JA, Horsewood P, Simpson PJ, Moore JC, Kelton JG. Impact of the patient population on the risk for heparininduced thrombocytopenia. Blood. 2000;96:1703-8.

7. Warkentin TE, Roberts RS, Hirsh J, Kelton JG. An improved definition of immune heparin-induced thrombocytopenia in postoperative orthopedic patients. Arch Intern Med. 2003;163:2518-24

8. El-Banayosy A, Korfer R, Arusoglu L, Kizner L, Morshuis M, Milting $\mathrm{H}$, et al. Device and patient management in a bridge-to-transplant setting. Ann Thorac Surg. 2001;71:S98-102; discussion S114-5.

9. Greinacher A, Michels I, Kiefel V, Mueller-Eckhardt C. A rapid and sensitive test for diagnosing heparin-associated thrombocytopenia. Thromb Haemost. 1991;66:734-6.

10. Greinacher A, Amiral J, Dummel V, Vissac A, Kiefel V, MuellerEckhardt C. Laboratory diagnosis of heparin-associated thrombocytopenia and comparison of platelet aggregation test, heparin-induced platelet activation test, and platelet factor 4/heparin enzyme-linked immunosorbent assay. Transfusion. 1994;34:381-5.

11. Koster A, Loebe M, Sodian R, Potapov EV, Hansen R, Muller J, et al. Heparin antibodies and thromboembolism in heparin-coated and noncoated ventricular assist devices. J Thorac Cardiovasc Surg. 2001; 121:331-5.

12. Dewald O, Fischlein T, Vetter HO, Schmitz C, Godje O, Gohring P, et al. Platelet morphology in patients with mechanical circulatory support. Eur J Cardiothorac Surg. 1997;12:634-41.

13. Houel R, Mazoyer E, Boval B, Kirsch M, Vermes E, Drouet L, et al. Platelet activation and aggregation profile in prolonged external ventricular support. J Thorac Cardiovasc Surg. 2004;128:197-202.

14. Untch B, Ahmad S, Jeske WP, Messmore HL, Hoppensteadt DA, Walenga JM, et al. Prevalence, isotype, and functionality of antiheparinplatelet factor 4 antibodies in patients treated with heparin and clinically suspected for heparin-induced thrombocytopenia. The pathogenic role of IgG. Thromb Res. 2002;105:117-23.

15. Hourigan LA, Walters DL, Keck SA, Dec GW. Heparin-induced thrombocytopenia: a common complication in cardiac transplant recipients. J Heart Lung Transplant. 2002;21:1283-9.

16. Fabris F, Luzzatto G, Soini B, Ramon R, Scandellari R, Randi ML, et al. Risk factors for thrombosis in patients with immune mediated heparin-induced thrombocytopenia. J Intern Med. 2002;252:149-54.

17. Chilver-Stainer L, Lammle B, Alberio L. Titre of anti-heparin/ PF4-antibodies and extent of in vivo activation of the coagulation and fibrinolytic systems. Thromb Haemost. 2004;91:276-82.

18. Warkentin TE, Kelton JG. Temporal aspects of heparin-induced thrombocytopenia. N Engl J Med. 2001;344:1286-92.

19. Greinacher A, Farner B, Kroll H, Kohlmann T, Warkentin TE, Eichler P. Clinical features of heparin-induced thrombocytopenia including risk factors for thrombosis. A retrospective analysis of 408 patients. Thromb Haemost. 2005;94:132-5.

20. Greinacher A, Eichler P, Lietz T, Warkentin TE. Replacement of unfractionated heparin by low-molecular-weight heparin for postorthopedic surgery antithrombotic prophylaxis lowers the overall risk of symptomatic thrombosis because of a lower frequency of heparininduced thrombocytopenia. Blood. 2005;106:2921-2.

21. Frazier OH, Rose EA, Oz MC, Dembitsky W, McCarthy P, Radovancevic $\mathrm{B}$, et al. Multicenter clinical evaluation of the HeartMate vented electric left ventricular assist system in patients awaiting heart transplantation. J Thorac Cardiovasc Surg. 2001;122:1186-95.

22. Navia JL, McCarthy PM, Hoercher KJ, Feng J, Jeevantham R, Smedira NG, et al. Do left ventricular assist device (LVAD) bridgeto-transplantation outcomes predict the results of permanent LVAD implantation? Ann Thorac Surg. 2002;74:2051-62; discussion 2062-3.

23. Eichler P, Budde U, Haas S, Kroll H, Loreth RM, Meyer O, et al. First workshop for detection of heparin-induced antibodies: validation of the heparin-induced platelet-activation test (HIPA) in comparison with a PF4/heparin ELISA. Thromb Haemost. 1999;81:625-9. 
24. Warkentin TE, Heddle NM. Laboratory diagnosis of immune heparininduced thrombocytopenia. Curr Hematol Rep. 2003;2:148-57.

25. Greinacher A, Warkentin TE. Recognition, treatment, and prevention of heparin-induced thrombocytopenia: review and update. Thromb Res. 2005;Aug 31. [Epub ahead of print.]

26. Merry AF. Bivalirudin, blood loss, and graft patency in coronary artery bypass surgery. Semin Thromb Hemost. 2004;30:337-46.

\section{Discussion}

Dr Harold L. Lazar (Boston, Mass). Twenty-five percent to $50 \%$ of all patients undergoing cardiac surgery will develop antibodies to heparin, but only $1 \%$ to $3 \%$ will actually develop the dreaded HIT syndrome. In their study, Dr Schenk and his colleagues have shown that HIT syndrome may be an important clinical syndrome in patients receiving MCS, who are routinely exposed to heparin before their operation. They have demonstrated that antiheparin antibodies developed in $35 \%$ of these patients and that these patients are at higher risk for early thromboembolic events. I have three questions for Dr Schenk and his colleagues.

First of all, what type of heparin did these patients receive? Previous studies have shown that the incidence of positive heparin antibodies can be reduced by as much as a third by using pork instead of beef heparin. In your study did you notice any difference between pork and beef heparin and the incidence of thromboembolic events?

Dr El-Banayosy. We are using pork heparin in all of our patients.

Dr Lazar. Second, in your study you used both the enzymelinked immunosorbent assay and the CAA to detect antiplatelet antibodies and thromboelastography to detect hypercoagulability. Since the decision to discontinue heparin is very important and the development of the thromboembolic event is devastating to these patients, on the basis of your data, what tests would you use to determine when heparin should be avoided, and would you recommend obtaining these tests in all patients before putting them on MCS devices?

Dr Schenk. We know that about 50\% of all patients have antibodies, but only $1 \%$ to $3 \%$ of those patients have the clinical HIT symptoms. We also know that there are several immunoglobulins, for example, IgG, IgA, and IgM, that are relevant, and, as you mentioned, those tests only assess the overall number of those antibodies. We cannot distinguish among $\operatorname{IgA}$, IgG, and $\operatorname{IgM}$ by the commercially available tests.

As to your point, we have to look for the presence of the clinically relevant antibodies, and we would also now add some additional testing. For example, we would add some platelet function tests, which can distinguish between those antibodies that are more pathogenic versus the others. That has been introduced now; unfortunately, we did not do that during the time frame of our study period.

The decision to discontinue heparin and to switch to another intravenous anticoagulant is based on an evaluation of all available laboratory tests together with clinical signs suggestive of HIT. If platelet counts have not recovered, oral anticoagulants should not be administered. If the patient no longer has low platelet counts, oral anticoagulants can be used as the clinical situation requires, and we do this with thromboelastogram guidance.

Dr Lazar. Finally, in view of your poor results with lepirudin and the platelet aggregate inhibitors, what therapeutic agents would you recommend for patients when heparin must be discontinued? Do you have any experience with bivalirudin (Angiomax), which seems to be used increasingly by our interventional cardiologists during angioplasty procedures for acute coronary syndromes?

Dr Schenk. Unfortunately, as of now we have not used the drug that you mentioned, but in our current patients we are using lepirudin, which is an analogous agent to bivalirudin. However, we did observe many side effects, and most of the time we are very concerned about bleeding complications. We observed many patients who did receive lepirudin and had bleeding complications.

When we have a patient with these antibodies, we try to give platelet stabilizing factors as soon as we can. For instance, there is one drug named dipyridamole (Persantine), and because that seems to stabilize platelet function, it may be especially useful in cases of HIT. In addition, we try to give aspirin and clopidogrel as well, which may have beneficial effects.

Dr Ralph Damiano (St Louis, Mo). Could you comment on your strategies in the patients who were HIT positive? I assume a number of these patients were bridged to transplant. Did you find a higher mortality at the time of the transplantation, and what were your strategies for managing the patients during that period?

Dr Schenk. The first part of your question concerns outcome related to survival. We did not see any difference in survival. Why that is, we do not know yet, but there was no difference in survival between antibody positive and negative patients.

The second part of your question concerns what we give if the patient has had HIT antibodies before. What do we do during transplant? HIT antibodies are transiently expressed. As long as you use heparin, the patient obviously is at risk. However, if you discontinue heparin, then those antibodies will likely disappear. If you re-evaluate those antibodies after 12 to 18 months when the patient is coming back for transplant, most of the time those antibodies are not effective anymore. Therefore, if you have a patient who had a history of HIT but has no current antibodies, there is no reason not to give heparin during cardiopulmonary bypass. Of course, you have to screen those antibodies closely, and as soon as antibodies come back, you had better give something else.

Dr Steven Bolling (Ann Arbor, Mich). Your unit uses many different types of circulatory support. Was there a different rate of HIT positivity associated with different ventricular assist or circulatory support devices? Were there heparin-bonded surfaces in these devices? The numbers may be small.

Dr Schenk. Thank you very much. This is a pretty important question because it emphasizes the pathology of that disease. We do not believe that this is device-related. Of course, we cannot prove that because we would have to have higher numbers and do a multivariable analysis. However, the only factor that is known to cause HIT is obviously heparin, and even if one device is found to be more related to HIT than the other, it is our belief that this is more treatment-related than device-related. I agree with you that HIT may be more prevalent with certain devices, but this should be related to the clinical indication for the device. For example, our patients who receive a CardioWest total artificial heart (SynCardia Systems, Inc, Tucson, Ariz) are among the sickest. They are usually receiving extracorporeal membrane oxygenation, they are usually in cardiogenic shock, and they are likely to be in the 
intensive care unit for some days to several weeks with heparin exposure as well. If you see a higher incidence of HIT positive patients, for example, with this particular device, then I think this is a matter of whether and how much heparin you gave before implant.

In addition, there is also reason to believe that there is an analogy to the HLA sensitization. There was a study done by the Cleveland Clinic last year. When they compared their HeartMate (Thoratec Corporation, Pleasanton, Calif) versus Novacor (WorldHeart Corporation, Oakland, Calif) data, they found that HLA sensitization was essentially not device-related; it was treatmentrelated, essentially a matter of the number of blood products used and not contingent on the device at all. So one thought I come back to is this: heparin is given before, during, and after surgery and therefore those antibodies may be produced in quite a number and with better specificity and for a longer time. I think it stands to reason that heparin is the only factor that causes HIT syndrome. On the other hand, since so much heparin is given, the likelihood of having HIT syndrome is probably among the highest in patients receiving MCS.

Dr El-Banayosy. I would like to make a short comment on that. Regarding the differences between the devices, we found that we had a higher incidence in the patients supported with the CardioWest total artificial heart. It might be due to the fact that the patients supported with a total artificial heart in our institution are the sickest cohort of patients and they have received heparin longer. But the numbers, as I mentioned, were not too high to allow us to see a statistically significant difference.

Dr Keith Allen (Indianapolis, Ind). I enjoyed your talk. Our group has had the opportunity to participate in some controlled trials using bivalirudin as an alternative to heparin. You suggested in one of your answers that in using lepirudin, which is one of the three direct thrombin inhibitors that are available, you had increased bleeding complications. I would like the audience to understand that the three direct thrombin inhibitors are not created equal. The problem you are having with lepirudin is related to its longer half-life and the inability to have any type of marker to measure the degree of anticoagulation. Bivalirudin has a shorter half-life and its anticoagulation can be measured. Therefore, I would argue that bivalirudin is a very attractive alternative to heparin in a patient with a left ventricular assist device.

Dr Eric A. Rose (New York, NY). There have been devices that have actually been designed to include a heparin coating on them. One such device that we have worked with is the MicroMed DeBakey device (MicroMed Technology, Inc, Houston, Tex). After review of outcomes looking at the heparin-coated versus the noncoated devices, we actually reached the conclusion that it offered no advantage and perhaps a disadvantage. Essentially, a device that is coated with heparin is almost potentially a heparin vaccine, and I think that is an important issue that needs to be part of device design.

Dr Schenk. Maybe I can add a dissenting point. In a study from the Berlin group done about 3 years ago, the Berlin Heart (Berlin Heart AG, Berlin, Germany), which is heparin-coated, was compared with other devices that are not. The Berlin group did not find any higher incidence of heparin antibodies between the groups with coated versus noncoated tubing. This actually would argue against the statement or against this idea that heparin coating is a bad thing to do. I think it is reasonable to say that heparin that is bound to a device may not be as immunogenic as heparin that is unfractionated and streams freely in the blood. 


\section{Appendix E1}

The following devices were used: CardioWest Total Artificial Heart (SynCardia Systems, Tucson, Ariz) $(\mathrm{n}=49)$, Thoratec Ventricular Assist Device (Thoratec Corporation, Pleasanton, Calif) $(\mathrm{n}=27)$, Novacor Left Ventricular Assist System (WorldHeart Corporation, Oakland, Calif) $(\mathrm{n}=20)$, Terumo DuraHeart Left Ventricular Assist System (Terumo Heart Incorporation, Ann Arbor, Mich) $(\mathrm{n}=10)$, Berlin Heart INCOR Ventricular Assist Device (Berlin Heart AG, Berlin, Germany) $(n=4)$, HeartMate VE Left Ventricular Assist System (Thoratec Corporation) ( $\mathrm{n}=$ 3), Medos HIA-Ventricular Assist Device (Medos, Stolberg, Germany) $(\mathrm{n}=2)$, Arrow LionHeart Left Ventricular Assist System (Arrow International, Reading, $\mathrm{Pa})(\mathrm{n}=2)$, Thoratec Implantable Ventricular Assist Device [IVAD] (Thoratec Corporation) $(\mathrm{n}=1)$, and the CorAide Left Ventricular Assist System (Arrow Interna- tional) $(\mathrm{n}=1)$. Four patients received multiple devices: a Thoratec, Novacor and CorAide devices were replaced by a CardioWest total artificial heart, and a HeartMate left ventricular assist device was aided by a Thoratec right ventricular assist device.

\section{Appendix E2}

Variables considered being associated with thrombotic events: anti-PF4 heparin antibodies, heparin-induced platelet activation, gender, age, device type, site of mechanical assistance/ replacement, aPTT, INR, fibrinogen, blood loss, packed red blood cells, fresh frozen plasma, units of platelets, surgical re-exploration, reoperation, preimplant continuous venovenous hemofiltration, preimplant extracorporeal membrane oxygenation, preimplant ventilation, preoperative intra-aortic balloon pump, etiology, and diabetes. 
TABLE E1. Patient characteristics

\begin{tabular}{|c|c|}
\hline Variable & $n=115$ \\
\hline \multicolumn{2}{|l|}{ Demography } \\
\hline Female & $22(19 \%)$ \\
\hline Age $(y ;$ mean $\pm S D)$ & $54.1 \pm 14.9$ \\
\hline Body surface area $\left(\mathrm{m}^{2} ;\right.$ mean $\left.\pm \mathrm{SD}\right)$ & $1.9 \pm 0.2$ \\
\hline \multicolumn{2}{|l|}{ Medical history } \\
\hline Ischemic cardiomyopathy & $54(47 \%)$ \\
\hline Dilated cardiomyopathy & $34(30 \%)$ \\
\hline Prior thoracic surgery & $40(35 \%)$ \\
\hline \multicolumn{2}{|l|}{ Preoperative support } \\
\hline Inotropes & $110(96 \%)$ \\
\hline IABP/ECMO & $60(52 \%)$ \\
\hline CVVH & $34(30 \%)$ \\
\hline Mechanical ventilation & $53(46 \%)$ \\
\hline \multicolumn{2}{|l|}{ Surgery and postoperative course } \\
\hline Blood loss* $(m L ;$ mean \pm SD [15th, 85th percentiles]) & $826 \pm 811[245,1545]$ \\
\hline Packed red blood cellst (units/10 days; mean \pm SD [15th, 85th percentiles]) & $14.6 \pm 11.1[4,26]$ \\
\hline Fresh frozen plasma† (units; mean \pm SD [15th, 85th percentiles]) & $5.7 \pm 8.9[0,12]$ \\
\hline Platelets $†$ (units; mean \pm SD [15th, 85th percentiles]) & $1.8 \pm 2.8[0,5]$ \\
\hline Re-exploration for bleeding & $40(35 \%)$ \\
\hline
\end{tabular}

$S D$, Standard deviation; $I A B P$, intra-aortic balloon pump; ECMO, extracorporal membrane oxygenation; $C V V H$, continuous venovenous hemofiltration. *Within 12 hours after surgery. $†$ Within 10 days after surgery.

TABLE E2. Anti-PF4/heparin antibodies, heparin-induced platelet activation, platelets, aPTT, and thrombotic events in HIT positive patients

\begin{tabular}{|c|c|c|c|c|c|c|c|c|c|c|c|c|c|c|}
\hline \multirow{2}{*}{$\begin{array}{l}\text { Pt. } \\
\text { No. }\end{array}$} & \multirow{2}{*}{$\begin{array}{l}\text { EIA } \\
\text { GTI }\end{array}$} & \multicolumn{3}{|c|}{ EIA HGW } & \multirow[b]{2}{*}{ HIPA } & \multicolumn{3}{|c|}{ aPTT } & \multicolumn{3}{|c|}{ Platelets } & \multirow[b]{2}{*}{ Event } & \multirow[b]{2}{*}{ POD } & \multirow[b]{2}{*}{ Treatment* } \\
\hline & & $\operatorname{Ig} G$ & $\operatorname{Ig} A$ & $\lg M$ & & POD 7 & POD 14 & POD 21 & POD 7 & POD 14 & POD 21 & & & \\
\hline 1 & 1.973 & 1.744 & 0.245 & 0.965 & pos & 54 & 45 & 89 & 251 & 662 & 211 & TIA & 14 & Not detected \\
\hline 2 & 1.215 & 1.562 & 0.330 & 0.350 & pos & 38 & 32 & 30 & 142 & 318 & 248 & TIA + CVA & $23 / 27$ & Epoprostenol \\
\hline 3 & 1.482 & 1.089 & 1.491 & 0.274 & pos & 35 & 40 & 50 & 371 & 416 & 353 & $\mathrm{TIA}$ & 21 & Lepirudin \\
\hline 4 & 0.839 & 1.032 & 0.256 & 0.507 & pos & 63 & 71 & 75 & 55 & 304 & 221 & CVA & 25 & Not detected \\
\hline 5 & 2.392 & 1.899 & 1.632 & 0.346 & pos & 49 & 33 & 30 & 79 & 270 & 213 & CVA & 25 & Epoprostenol \\
\hline 6 & 1.599 & 1.804 & 0.464 & 0.659 & pos & 51 & 53 & 45 & 37 & 89 & 262 & None & & Not detected \\
\hline 7 & 0.503 & 1.254 & 0.273 & 0.282 & id & 55 & 59 & 54 & 90 & 218 & 217 & None & & Not detected \\
\hline 8 & 2.291 & 1.915 & 1.361 & 0.671 & pos & 47 & 33 & 30 & 56 & 64 & 139 & TIA + CVA & $11 / 12$ & ASA/phenprocoumon \\
\hline 9 & 0.485 & 0.716 & 0.447 & 0.453 & pos & 53 & 38 & 41 & 68 & 59 & 34 & Limb ischemia & 11 & Lepirudin \\
\hline 10 & 2.212 & 1.913 & 1.726 & 0.385 & pos & 63 & 46 & 57 & 40 & 168 & 205 & CVA & 24 & Not detected \\
\hline 11 & 2.024 & 1.819 & 0.46 & 0.700 & pos & 47 & 42 & 34 & 47 & 166 & 214 & TIA & 30 & Lepirudin \\
\hline 12 & 1.604 & 1.320 & 1.777 & 1.746 & id & 64 & 41 & 61 & 53 & 54 & 184 & CVA & 21 & Not detected \\
\hline
\end{tabular}

EIA GTI, Anti-PF/heparin antibody by polyspecific enzyme immunoassay (titer); EIA HGW, anti-PF/heparin antibody classes by monospecific enzyme immunoassay (titer); HIPA, status of heparin-induced platelet activation (positive/indeterminate [pos/id]); aPTT, activated partial thromboplastin time; POD, postoperative day; $T I A$, transient ischemic attack; CVA, cerebrovascular accident. *Treatment with alternative anticoagulation on diagnosis during hospital stay. Note that in 6 patients the condition remained undetected during their hospital stay, resulting in their receiving heparin. 
TABLE E3. Standard coagulation monitoring

\begin{tabular}{|c|c|c|c|c|c|c|}
\hline & POD 3 & POD 7 & POD 10 & POD 14 & POD 21 & POD 28 \\
\hline Fibrinogen $(n)^{*}$ & $(12 / 28 / 73)$ & $(11 / 28 / 69)$ & $(10 / 24 / 64)$ & (11/17/48) & $(8 / 14 / 36)$ & $(6 / 9 / 32)$ \\
\hline HIPA+\&id/lgG + & $575(470 ; 691)$ & $618(521 ; 729)$ & $660(555 ; 765)$ & $511(417 ; 606)$ & $566(475 ; 657)$ & $586(439 ; 739)$ \\
\hline HIPA $-/ \lg G, A, M+$ & $553(484 ; 624)$ & $614(565 ; 663)$ & $614(549 ; 678)$ & $573(476 ; 670)$ & $571(467 ; 676)$ & $567(433 ; 700)$ \\
\hline HIT neg & $537(501 ; 573)$ & $568(523 ; 612)$ & $569(524 ; 614)$ & 587 (526; 648) & $536(465 ; 607)$ & $514(449 ; 579)$ \\
\hline$P$ & .721 & .371 & .218 & .523 & .810 & .538 \\
\hline $\operatorname{DDIM}(n)^{*}$ & $(12 / 24 / 62)$ & $(12 / 28 / 71)$ & & & & \\
\hline HIPA+\&id/lgG + & $1004(519 ; 1488)$ & $1183(563 ; 1802)$ & & & & \\
\hline $\mathrm{HIPA}-/ \lg \mathrm{G}, \mathrm{A}, \mathrm{M}+$ & $898(533 ; 1262)$ & $1460(827 ; 2092)$ & & & & \\
\hline HIT neg & $694(566 ; 829)$ & $843(645 ; 1041)$ & & & & \\
\hline$P$ & .189 & .042 & & & & \\
\hline $\operatorname{INR}(n)^{*}$ & $(12 / 28 / 73)$ & $(11 / 28 / 73)$ & $(11 / 24 / 69)$ & $(12 / 21 / 62)$ & $(11 / 21 / 51)$ & $(9 / 17 / 40)$ \\
\hline HIPA + \&id/lgG + & $1.12(1.00 ; 1.25)$ & $1.47(0.96 ; 1.99)$ & $1.66(0.93 ; 2.40)$ & $1.52(1.06 ; 1.98)$ & $1.67(1.10 ; 2.24)$ & $2.01(1.29 ; 2.74)$ \\
\hline HIPA $-/ \lg G, A, M+$ & $1.44(1.11 ; 1.77)$ & $1.50(1.20 ; 1.79)$ & $1.34(1.11 ; 1.56)$ & $1.28(1.13 ; 1.42)$ & $1.41(1.18 ; 1.65)$ & $1.42(1.17 ; 1.67)$ \\
\hline HIT neg & $1.38(1.23 ; 1.53)$ & $1.39(1.24 ; 1.54)$ & $1.30(1.20 ; 1.40)$ & $1.29(1.19 ; 1.39)$ & $1.48(1.24 ; 1.71)$ & $1.70(1.41 ; 1.99)$ \\
\hline$P$ & .394 & .774 & .131 & .233 & .665 & .205 \\
\hline aPTT $(n)^{*}$ & $(12 / 28 / 73)$ & $(12 / 28 / 73)$ & $(11 / 23 / 70)$ & $(12 / 21 / 60)$ & $(10 / 21 / 52)$ & $(9 / 17 / 40)$ \\
\hline HIPA+\&id/lgG + & $42(35 ; 49)$ & $46(38 ; 54)$ & $47(37 ; 56)$ & $46(38 ; 55)$ & $47(35 ; 60)$ & $45(35 ; 54)$ \\
\hline HIPA-/IgG,A,M+ & $44(40 ; 47)$ & $51(54 ; 56)$ & $53(46 ; 59)$ & $47(42 ; 52)$ & $51(45 ; 57)$ & $56(36 ; 75)$ \\
\hline HIT neg & $45(42 ; 47)$ & $51(46 ; 56)$ & $48(45 ; 51)$ & $51(48 ; 54)$ & $51(47 ; 55)$ & $51(45 ; 56)$ \\
\hline$P$ & .749 & .754 & .303 & .295 & .784 & .516 \\
\hline
\end{tabular}

POD, Postoperative day; DDIM, D-dimers; INR, international normalized ratio; aPTT, activated partial thromboplastin time; HIPA+\&id/IgG+, heparininduced platelet activation assay positive or indeterminate with IgG titers of $O D>1.0 ; H I T-$, heparin-induced thrombocytopenia negative; HIPA $A_{N E G}$ $\lg G, A, M+$, heparin-induced thrombocytopenia platelet activation assay negative and $\lg \mathrm{G}$, $\lg \mathrm{A}$, and $\lg \mathrm{M}$ antibodies. *Number of patients for whom data are available (HIPA+\&id/lgG+/ HIPA-/IgG,A,M+/HIT negative).

TABLE E4. Classification table for multivariate regression model*

\begin{tabular}{|c|c|c|c|c|}
\hline \multirow[b]{3}{*}{ Observed } & \multicolumn{4}{|c|}{ Predicted } \\
\hline & \multicolumn{2}{|l|}{ Selected cases $\dagger$} & \multicolumn{2}{|c|}{ Unselected cases $†$} \\
\hline & $\begin{array}{l}\text { Thrombotic events } \\
\text { within } 2 \text { months }\end{array}$ & $\%$ correct & $\begin{array}{l}\text { Thrombotic events } \\
\text { within } 2 \text { months }\end{array}$ & $\%$ correct \\
\hline \multicolumn{5}{|c|}{ Thrombotic events within 2 months } \\
\hline No & 41 & 93.2 & 30 & 83.3 \\
\hline Yes & 14 & 73.7 & 6 & 60.0 \\
\hline Overall \% & & 87.3 & & 76.5 \\
\hline
\end{tabular}

*Forward stepwise inclusion of all variables. Variables were retained at $P<.05$. Similar results were obtained by backward stepwise elimination of variables (data not shown). †Bernoulli distribution. 


\section{ROC-Curve}

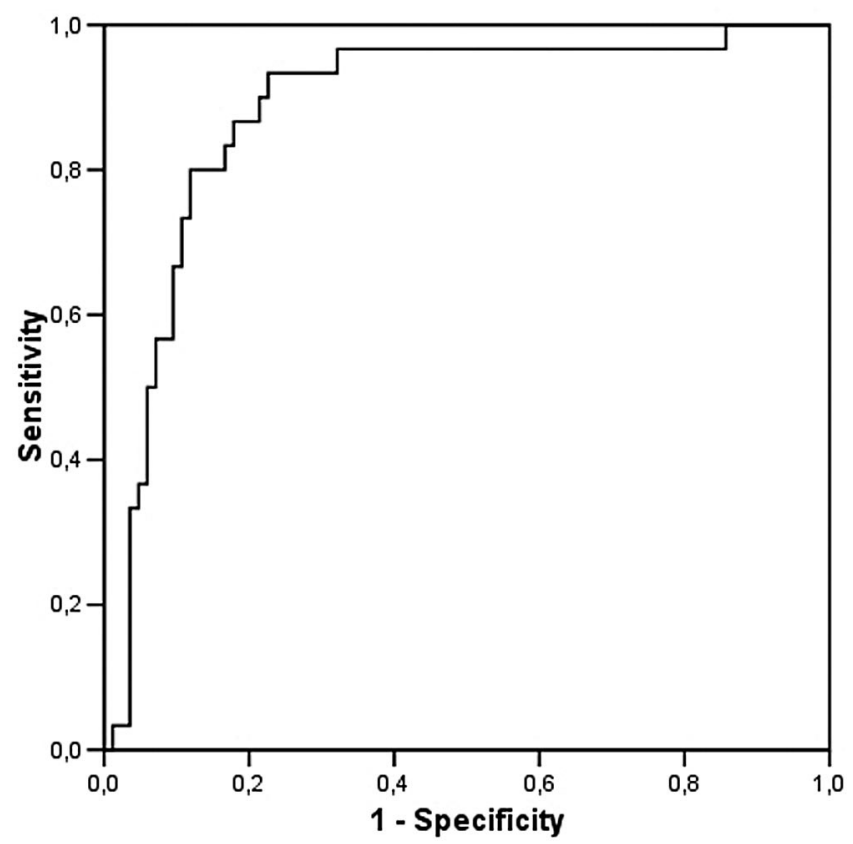

Figure E1. Receiver operating characteristic (ROC) curve for multivariate regression model to predict thrombotic events. Area under curve $0.885 \pm 0.037(0.813 ; 0.956) ; P<.001$. Regression equation: $z=-9.017+$ $2.454 \cdot b_{1}+4.292 \cdot b_{2}+0.009 \cdot b_{3}$, where $b_{1}$ is titer of anti-PF4/heparin antibodies by polyspecific enzyme immunoassay (OD), $b_{2}$ is gender $\left(1\right.$, female; 0 , male), and $b_{3}$ is fibrinogen $(\mathrm{mg} / \mathrm{dL})$.

Table E5. Predictive capabilities of anti-PF4/heparin antibody tests for thromboembolic events

\begin{tabular}{|c|c|c|c|c|c|}
\hline & $\begin{array}{c}\text { EIA GTI } \\
\text { (OD > 0.5) }\end{array}$ & $\begin{array}{c}\text { EIA GTI } \\
(\mathrm{OD}>1.0)\end{array}$ & $\begin{array}{c}\text { EIA GTI (OD > } 0.5) \\
\text { or } \mathrm{CAA} \geq 1\end{array}$ & $\begin{array}{c}\text { EIA GTI (OD > 0.5) } \\
\text { or CAA } \geq 1 \text { and } \\
\operatorname{IgG}(\mathrm{OD}>1.0)\end{array}$ & $\begin{array}{c}\text { EIA GTI (OD > 0.5) } \\
\text { or CAA } \geq 1 \text { and } \\
\text { HIPA positive }\end{array}$ \\
\hline Sensitivity & 0.69 & 0.42 & 0.81 & 0.38 & 0.31 \\
\hline Specificity & 0.69 & 0.93 & 0.51 & 0.84 & 0.97 \\
\hline Predictive value & 0.52 & 0.75 & 0.44 & 0.73 & 0.90 \\
\hline Positive (95\% Cl) & $(0.41 ; 0.61)$ & $(0.53 ; 0.90)$ & $(0.36 ; 0.49)$ & $(0.48 ; 0.91)$ & $(0.57 ; 0.99)$ \\
\hline Predictive value & 0.83 & 0.77 & 0.84 & 0.65 & 0.64 \\
\hline Negative $(95 \% \mathrm{CI})$ & $(0.74 ; 0.89)$ & $(0.72 ; 0.80)$ & $(0.73 ; 0.93)$ & $(0.57 ; 0.70)$ & $(0.58 ; 0.66)$ \\
\hline$P$ & $<.001$ & $<.001$ & .004 & .021 & .005 \\
\hline
\end{tabular}

EIA GTI, Anti-PF/heparin antibody by polyspecific enzyme immunoassay (OD- titer); HIPA, status of heparin-induced platelet activation (positive/negative); $C A A$, column agglutination assay (grade $0-3$ ); $I g$, immunoglobulin; $C l$, confidence interval. 